\title{
Multiple Phase Anomalies in Bessel Beam
}

\author{
Myun-Sik Kim, ${ }^{1, *}$ Toralf Scharf, ${ }^{1}$ Alberto C. Assafrao, ${ }^{2}$ Silvania F. Pereira, ${ }^{2}$ \\ H. Paul Urbach, ${ }^{2}$ and Hans Peter Herzig ${ }^{1}$ \\ ${ }^{1}$ Optics \& Photonics Technology Laboratory, Ecole Polytechnique Fédérale de Lausanne (EPFL) Breguet 2, 2000 Neuchâtel, \\ Switzerland \\ ${ }^{2}$ Optics Research Group, Delft University of Technology, Lorentzweg 1, 2628 CJ Delft, The Netherlands \\ *Corresponding author: myunsik.kim@epfl.ch
}

\begin{abstract}
The non-diverging feature of the Bessel-kind beam leads to multiple phase anomalies along the optical axis in contrary to the focused Gaussian beam. Here, we analyze the phase distributions using in a longitudinal-differential phase mode of a high-resolution interference microscope. The measured data are compared with rigorous simulations, showing an excellent agreement.

OCIS Codes: (260.6042) Singular optics; (050.5080) Phase shift; (120.5050) Phase measurement.
\end{abstract}

\section{Introduction}

In 1890, Gouy discovered a peculiar axial phase shift of a convergent optical beam when passing through a focus. This phenomenon was later known as Gouy phase or a phase anomaly [1-3]. In his original experiments [1], the light diverging from a point source was reflected from a planar and a concave mirror resulting in two light beams that were overlapped in order to interfere. In this scheme circular fringes appear and the central fringe was found to change from dark to bright, or vice versa, at observation planes just before or after the focal point. This change of intensity indicates a $\pi$ phase shift when passing through the focus [3]. Such a phase anomaly has been observed in various types of focused beams, for instance, in Laguerre-Gaussian (LG) beams [4], radially polarized beams [5], in Airy beam [6], and in non-diffracting Bessel beams [7]. In addition to the highly focused optical beams, surface plasmon-polaritons [8], matter waves [9], a scattered hotspot (i.e. a photonic nanojet) [10], and a diffracted hotspot (i.e. the spot of Arago) [11] also exhibit the Gouy phase or comparable phase anomalies.

In this work, we experimentally and theoretically investigate how the non-diverging feature of the Bessel beam influences on the phase anomaly. Longitudinal-differential interferometry [11] allows us to directly measure the axial phase shifts, i.e. the phase anomaly, of the Bessel beam. Rigorous simulations provide the numerical counterparts, which verify the experimental results.

\section{Experiments and simulations}

A high-resolution interference microscope (HRIM) allows for measuring the amplitude and phase in the entire three-dimensional (3D) object space. The details of the experimental setup, with exemplary micro-optical elements, are reported in our previous works [see Ref. 11 and the references therein]. All experimental and numerical investigations were performed at a single wavelength of $642 \mathrm{~nm}$ (CrystaLaser: DL640-050-3). When the central part of a plane-wave illumination is blocked by an annular aperture, which is inserted at the entrance pupil of the focusing lens (Leica Microsystem, 50X/NA0.9 HXC PL APO), the amplitude distribution in the focal plane resembles a zeroth-order Bessel function of the first kind in radial direction within a finite extension [12]. Focusing causes a Gaussian apodization in the amplitude distribution that can be represented as a product of a Bessel function and a Gaussian profile. This type of experimental light beam is known as Bessel-Gauss beam [13]. Unlike a diffraction-free beam in theory [14], such beams carry a finite power and finite transverse and longitudinal extensions due to the fast decrease of the Gaussian profile. The longitudinal-differential phase mode of the HRIM records the phase difference of such a beam compared to that of a plane wave. This longitudinal-differential phase distribution visualizes the amount of the axial phase shift. Moreover, the propagation phase distribution can be retrieved by wrapping the longitudinal phase with a known value, for instance, the wavelength. In order to simulate such an experimental situation, the Debye diffraction integral is solved using the Richard and Wolf method [15] to perform vectorial aplanatic focusing of high NA lenses. By filtering out the inner NA part the central blockage of the annular aperture is simulated. This perfectly mimics the experimental situations and provides comparable results.

\section{Results and discussions}

The annular aperture, with $70 \%$ of central blocking of the entrance pupil, will cover the numerical aperture smaller than an equivalent angle of $55^{\circ}, \mathrm{NA}<0.82$. An example of the measured amplitude and phase distributions of the generated BesselGauss beam in the $x-z$ plane is shown in Fig. 1. The amplitude distribution in Fig. 1(a) exhibits the prominent intensity and the elongated focal spot along the z-axis, which are the typical features of the Bessel-kind beams. The measured longitudinaldifferential phase map is shown in Fig. 1(b) whilst Fig. 1(c) shows the propagation phase map retrieved by wrapping the phase with the effective wavelength along the central lobe $\left[\lambda_{\mathrm{eff}}=\lambda_{\text {air }} / \cos 55^{\circ}=1.1 \mu \mathrm{m}\right]$. The phase anomaly of the Bessel-Gauss beam is found to be very different from that of the focused Gaussian beam. The phase shift is not limited to $\pi$ (i.e. general Gouy phase 
for a point focusing) but multiples of $\pi$. Depending on the maximum angle of the blocking and the propagation length of the central lobe, it could be fractional multiples of $2 \pi$ and not only integer multiples. In the case of Fig. 1(b), the phase anomaly within the central lobe is found to be approximately $6 \pi$. The non-diverging character of the Bessel beam leads to axial phase shifting larger than $\pi$ and the phase shift (anomaly) continues to increase until the end of the propagation length.

Simulations are performed to prove our experimental findings. The amplitude and phase of the focused electric field is computed within a finite domain in the vicinity of the focal plane. By blocking the central NA, one can create light fields representing the experimental situations for the Bessel-Gauss beam of Fig. 1. Figure 2 shows the simulated amplitude and phase distributions of the Bessel-Gauss beam in the $x$-z plane. Here, the calculated phase field is the propagation phase shown in Fig. 2(c), and the longitudinal-differential phase in Fig. 2(b) is obtained by comparing this to the phase of a plane wave of the same wavelength $\left(\lambda_{\text {air }}\right.$ $=642 \mathrm{~nm}$ ). They show an excellent agreement with the experimental results. To the best of our knowledge, these are the first experimental visualization of such multiple phase anomalies of the Bessel-Gauss beam, supported by numerical counterparts. In our contribution we will give more details of physical meanings of such peculiar phase behaviors of non-diverging beams.
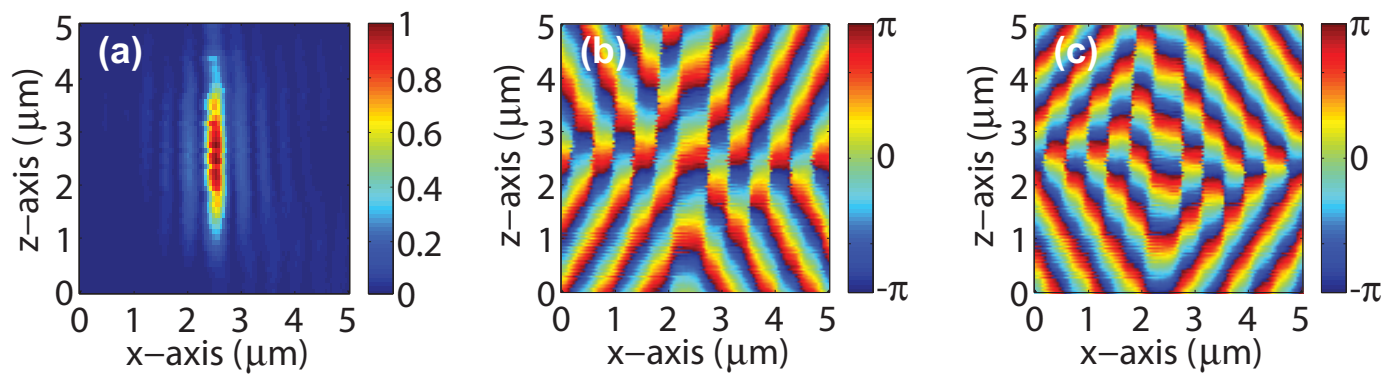

Fig. 1. Measured amplitude and phase distributions in the $x$-z plane: (a) amplitude, (b) longitudinal-differential phase and (c) propagation phase. The intensity is normalized. The phase is displayed in radian. The image size is $5 \times 5 \mu \mathrm{m}^{2}$.
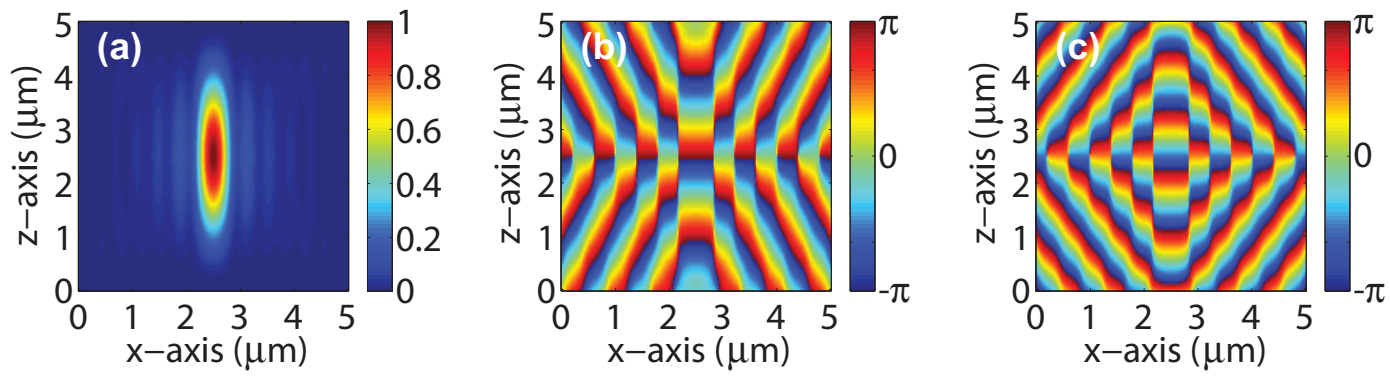

Fig. 2. Simulated amplitude and phase distributions in the $x$-z plane: (a) amplitude, (b) longitudinal-differential phase and (c) propagation phase. The intensity is normalized. The phase is displayed in radian. The image size is $5 \times 5 \mathrm{~m}^{2}$.

\section{References}

[1] L. G. Gouy, "Sur une propriété nouvelle des ondes lumineuses," C. R. Hebdomadaires Séances Acad. Sci. 110, 1251 (1890).

[2] T. D. Visser and E. Wolf, "The origin of the Guoy phase anomaly and its generalization to astigmatic wavefields", Opt. Commun. 283, 3371 (2010).

[3] A. E. Siegman, Lasers (University Science books, 1986). Note that Gouy's name is misspelled as “Guoy".

[4] J. Hamazaki, Y. Mineta, K. Oka, and R. Morita, "Direct observation of Gouy phase shift in a propagating optical vortex,” Opt. Express 14, 8382 (2006).

[5] H. Chen, Q. Zhan, Y. L. Zhang, and Y. P. Li, "The Gouy phase shift of the highly focused radially polarized beam," Phys. Lett. A 371, 259 (2007).

[6] X. Pang, G. Gbur, and T. D. Visser, "The Gouy phase of Airy beams," Opt. Lett. 36, 2492 (2011).

[7] P. Martelli, M. Tacca, A. Gatto, G. Moneta, and M. Martinelli, “Gouy phase shift in nondiffracting Bessel beams," Opt. Express 18, 7108 (2010).

[8] W. Zhu, A. Agrawal, and A. Nahata, "Direct measurement of the Gouy phase shift for surface plasmon-polaritons," Opt. Express 15, 9995 (2007).

[9] I. G. da Paza, M. C. Nemes, S. Pádua, C. H. Monken, and J. G. Peixoto de Faria, "Indirect evidence for the Gouy phase for matter waves," Phys. Lett. A 374, 1660 (2010).

[10] M.-S. Kim, T. Scharf, S. Mühlig, C. Rockstuhl, and H. P. Herzig, "Gouy Phase Anomaly in Photonic Nanojets,” Appl. Phys. Lett. 98, 191114 (2011).

[11] M.-S. Kim, T. Scharf, C. Etrich, C. Rockstul, and H. P. Herzig, "Longitudinal-differential interferometry: Direct imaging of axial superluminal phase propagation," Opt. Lett. 37, 305 (2012).

[12] M. Born and E. Wolf, Principles of Optics (Cambridge University Press, 1999), $7^{\text {th }}$ ed.

[13] F. Gori, G. Guattari, and C. Padovani, "Bessel-Gauss beams," Optics Comm. 64, 491 (1987).

[14] J. Durnin, J.J. Miceli and J.H. Eberly, "Diffraction-free beams," Phys. Rev. Lett. 58, 1499 (1987).

[15] B. Richards and E. Wolf, "Electromagnetic diffraction in optical systems, II Structure of the image field in an Optical system," Proc. R. Soc. Lond. A. 253(1274), 358 (1959).

\section{Acknowledgements}

The research leading to these results has received funding from the European Space Agency. 